Elect. Comm. in Probab. 8 (2003) 1-5

\title{
THE MEAN OF A MAXIMUM LIKELIHOOD ESTIMA- TOR ASSOCIATED WITH THE BROWNIAN BRIDGE
}

\section{FUCHANG GAO}

Department of Mathematics

University of Idaho

Moscow, ID 83844-1103

(Research partially supported by NSF grant EPS-0132626)

email: fuchang@uidaho.edu

Submitted 18 March 2002, accepted in final form 15 Jan 2003

AMS 2000 Subject classification: 60G15 (52A07 52A22 52A39 60D05 60E15)

Keywords: Brownian bridge, intrinsic volume, solid angle

\section{Abstract}

A closed formula for the mean of a maximum likelihood estimator associated with the Brownian bridge is obtained; the exact relation with that of the Brownian motion is established.

\section{Introduction}

Let $X_{t}, t \in T$, be a Gaussian process, and $\mu$ be a probability measure on $T$. To avoid measurability questions, we take $T$ as finite. Denote $X_{\mu}=\int_{T} X_{t} \mu(d t)$. Consider the following maximum likelihood estimator

$$
\sup _{\mu} \exp \left(\lambda X_{\mu}-\frac{\lambda^{2}}{2} \mathbb{E}\left|X_{\mu}\right|^{2}\right),
$$

where the supremum is taken over all the probability measures on $T$. This maximum likelihood estimator (introduced in a slightly different way) has been studied extensively (c.f. [3]-[5]). By taking the $\delta$ measure at $t \in T$, it is easy to see that (1) dominates

$$
\sup _{t \in T} \exp \left(\lambda X_{t}-\frac{\lambda^{2}}{2} \mathbb{E}\left|X_{t}\right|^{2}\right) \text {. }
$$

Thus, there is a close connection between (1) and the supremum of the Gaussian process. However, because of the quadratic term $\frac{\lambda^{2}}{2} \mathbb{E}\left|X_{\mu}\right|^{2}$ in (1), there seems to be no direct derivation from one to the other. In particular, what is the mean

$$
W_{X}(\lambda)=: \mathbb{E} \sup _{\mu} \exp \left(\lambda X_{\mu}-\frac{\lambda^{2}}{2} \mathbb{E}\left|X_{\mu}\right|^{2}\right)
$$

for a given Gaussian process? (The use of the letter "W" will become clear later, once the connection with the Wills functional is established.) By omitting the quadratic term, it is 
clear that (3) is bounded above by the moment generating function of $\sup _{t \in T} X_{t}$. Note that the exact expression for the moment generating function is hard to obtain (in fact, it is known only for six special Gaussian processes). Thus, (3) is an alternative quantity to study. We believe that (3) contains rich information about a Gaussian process. For example, by studying its upper bound, [6] obtained an exponential inequality, which is stronger than the well-known deviation inequality for the supremum of a Gaussian process.

Motivated by this, we consider (3) for Brownian bridge on [0,1]. We choose this process because of the nice geometric structure, and more importantly, because the moment generating function of the supremum is known. This allows one to study the connections between the two. It turns out that for Brownian bridge, (3) is of very nice closed form.

Theorem 1 If $M_{t}$ and $B_{t}$ are the Brownian motion and the Brownian bridge on [0,1] respectively, then

$$
W_{B}(\lambda)=: \mathbb{E} \sup _{\mu} \exp \left(\lambda B_{\mu}-\frac{\lambda^{2}}{2} \mathbb{E}\left|B_{\mu}\right|^{2}\right)=\frac{1}{2} \sum_{k=0}^{\infty} \frac{\omega_{k+1}}{k !}\left(\frac{\lambda}{\sqrt{2 \pi}}\right)^{k}
$$

and

$$
W_{M}(\lambda)=: \mathbb{E} \sup _{\mu} \exp \left(\lambda M_{\mu}-\frac{\lambda^{2}}{2} \mathbb{E}\left|M_{\mu}\right|^{2}\right)=1+\frac{2}{\sqrt{2 \pi}} \int_{0}^{\lambda} W_{B}(t) d t
$$

where the supremum is over all the probability measure on $[0,1]$, and $\omega_{k}=\pi^{k / 2} / \Gamma(k / 2+1)$ is the volume of the $k$-dimensional unit ball.

(5) should be compared with the relation between the moment generating functions $\mathcal{M}_{M}(\lambda)$ and $\mathcal{M}_{B}(\lambda)$ of the suprema of Brownian motion and Brownian bridge:

$$
\mathcal{M}_{M}(\lambda)=1+\frac{2}{\sqrt{2 \pi}} \int_{0}^{\lambda} \mathcal{M}_{B}(2 t) d t
$$

It is interesting that there is an extra factor 2 in $\mathcal{M}_{B}$.

\section{Connecting to geometry}

By approximation and continuity, we can assume $T$ consist of $n+1$ elements. Equipped with the distance $\operatorname{dist}\left(X_{t}, X_{s}\right)=: \sqrt{\mathbb{E}\left|X_{t}-X_{s}\right|^{2}},\left\{X_{t}: t \in T\right\}$ can be viewed as a set in $\mathbb{R}^{n}$. Let $K$ be the convex hull of this set. Then for any probability measure $\mu$ on $T, X_{\mu}$ is a point in $K$. It is then not hard to derive that

$$
\mathbb{E} \sup _{\mu} \exp \left(\lambda X_{\mu}-\frac{\lambda^{2}}{2} \mathbb{E}\left|X_{\mu}\right|^{2}\right)=\int_{\mathbb{R}^{n}} \exp \left(-\pi \operatorname{dist}^{2}(x, \lambda K / \sqrt{2 \pi})\right) d x .
$$

(See [6]). The right hand side is usually called the Wills functional of $K$, and can be expressed as

$$
\sum_{k=0}^{\infty} V_{k}(K)\left(\frac{\lambda}{\sqrt{2 \pi}}\right)^{k}
$$

where $V_{k}(K)$ is the $k^{\text {th }}$ intrinsic volume of $K$. (See [1] or [7]). It is known that $V_{k}(K)$ can be evaluated by

$$
V_{k}(K)=\sum_{F_{J}}\left|F_{J}\right| \gamma\left(N\left(F_{J}, K\right)\right)
$$


where $\left|F_{J}\right|$ is the Lebesgue measure of the $k$-dimensional face $F_{J}$ of $K, \gamma\left(N\left(F_{J}, K\right)\right)$ is the Gaussian measure of the solid angle of the normal cone $N\left(F_{J}, K\right)$ at $F_{J}$, and the sum is over all the $k$-dimensional faces of $K$.

Thus the evaluation of $W_{X}(\lambda)$ becomes a problem of computing solid angles.

\section{Proof of the theorem}

Because there is no formula available in general on the evaluation of higher dimensional solid angles, from now on, we restrict ourselves to the Brownian bridge setting.

A Brownian bridge on $[0,1]$ is a Gaussian process $B_{t}(\omega), 0 \leq t \leq 1$, with covariance $\mathbb{E}\left(B_{s} B_{t}\right)=$ $\min (s, t)-s t$ for $s, t \in[0,1]$. For any $1 \leq T \leq 1$, consider the following special discretization of $B_{t}, 0 \leq t \leq T$. For positive integer $n$, let $m$ be the largest integer such that $m / n \leq T$. View $\{0, B(1 / n), B(2 / n), \ldots, B(m / n)\}$ as $m+1$ points in $\mathbb{R}^{m}$, whose convex hull is an $m$ dimensional simplex. Up to scaling by $\sqrt{n}$, this simplex has the vertices $P_{0}=(0,0, \ldots, 0)$, and $P_{i}=(\underbrace{1, \cdots, 1}_{i}, 0, \ldots, 0)-\frac{i}{n}(1,1, \cdots, 1), 1 \leq i \leq m$. One can view it as the simplex with vertices $Q_{0}=(0,0, \cdots)$, and $Q_{i}=(\underbrace{1, \cdots, 1}_{i}, 0, \ldots, 0), 1 \leq 1 \leq m$, having each vertex twisted and pulled back in the "diagonal" direction. Denote this simplex by $K_{n}$.

For $1 \leq k<m$, let $J=\left\{i_{0}, i_{1}, i_{2}, \ldots, i_{k}\right\}$ be a set of integers, such that $0 \leq i_{0}<i_{1}<i_{2}<$ $\cdots<i_{k} \leq m$. Let $F_{J}$ be the $k$-dimensional face of $K$ that contains $P_{i}, i \in J$, and $\left|F_{J}\right|$ be the $k$-volume of $F_{J}$.

\section{Lemma 1}

$$
\left|F_{J}\right|=\frac{1}{k !} \sqrt{\left(1-\frac{i_{k}-i_{0}}{n}\right)\left(i_{k}-i_{k-1}\right) \times\left(i_{k-1}-i_{k-2}\right) \times \cdots \times\left(i_{1}-i_{0}\right)} .
$$

Proof: Let $D_{J}$ be the volume of the parallelepiped body generated by the vectors ${\overrightarrow{P_{0}}}_{P_{j}}, j \in J$, $j \neq i_{0}$. Then $\left|F_{J}\right|=D_{J} / k$ !. Note $D_{J}$ is just the square root of the determinant of the $k \times k$ symmetric matrix $Z_{J}=\left(z_{s t}\right)$, where $z_{s t}$ is the inner product of $\vec{P}_{i_{0} P_{i_{s}}}$ and $\vec{P}_{i_{0} P_{i_{t}}}, 1 \leq s \leq k$, $1 \leq t \leq k$. A direct calculation gives $z_{s t}=n^{-1}\left(i_{s}-i_{0}\right)\left[n-\left(i_{t}-i_{0}\right)\right]$ for $0<s \leq t \leq k$. Denote $d_{l}=i_{l}-i_{0}$. Then

$$
\operatorname{det}\left(Z_{J}\right)=\left|\begin{array}{lllll}
n^{-1} d_{1}\left(n-d_{1}\right) & n^{-1} d_{1}\left(n-d_{2}\right) & \cdots & n^{-1} d_{1}\left(n-d_{k-1}\right) & n^{-1} d_{1}\left(n-d_{k}\right) \\
n^{-1} d_{1}\left(n-d_{2}\right) & n^{-1} d_{2}\left(n-d_{2}\right) & \cdots & n^{-1} d_{2}\left(n-d_{k-1}\right) & n^{-1} d_{2}\left(n-d_{k}\right) \\
\cdots & \cdots & \cdots & \cdots & \cdots \\
n^{-1} d_{1}\left(n-d_{k-1}\right) & n^{-1} d_{2}\left(n-d_{k-1}\right) & \cdots & n^{-1} d_{k-1}\left(n-d_{k-1}\right) & n^{-1} d_{k-1}\left(n-d_{k}\right) \\
n^{-1} d_{1}\left(n-d_{k}\right) & n^{-1} d_{2}\left(n-d_{k}\right) & \cdots & n^{-1} d_{k-1}\left(n-d_{k}\right) & n^{-1} d_{k}\left(n-d_{k}\right)
\end{array}\right|
$$

Subtracting row $k$ by $\frac{n-d_{k}}{n-d_{k-1}}$ multiple of row $(k-1)$, and expanding along row $k$, we obtain

$$
\left(d_{k}-d_{k-1}\right) \frac{n-d_{k}}{n-d_{k-1}}\left|\begin{array}{llll}
n^{-1} d_{1}\left(n-d_{1}\right) & n^{-1} d_{1}\left(n-d_{2}\right) & \cdots & n^{-1} d_{1}\left(n-d_{k-1}\right) \\
n^{-1} d_{1}\left(n-d_{2}\right) & n^{-1} d_{2}\left(n-d_{2}\right) & \cdots & n^{-1} d_{2}\left(n-d_{k-1}\right) \\
\cdots & \cdots & \cdots & \cdots \\
n^{-1} d_{1}\left(n-d_{k-1}\right) & n^{-1} d_{2}\left(n-d_{k-1}\right) & \cdots & n^{-1} d_{k-1}\left(n-d_{k-1}\right)
\end{array}\right|
$$

Continuing the procedure, we finally obtain 


$$
\begin{aligned}
\operatorname{det}\left(Z_{J}\right) & =n^{-1}\left(n-d_{k}\right)\left(d_{k}-d_{k-1}\right) \cdots\left(d_{2}-d_{1}\right) d_{1} \\
& =n^{-1}\left[n-\left(i_{k}-i_{0}\right)\right]\left(i_{k}-i_{k-1}\right) \cdots\left(i_{1}-i_{0}\right)
\end{aligned}
$$

Lemma 1 follows.

In order to evaluate the $k^{\text {th }}$ intrinsic volume of $K$, we need to compute the Gaussian measure of the normal cone $N\left(F_{J}, K\right)$ at $F_{J}$. For $1 \leq j \leq m+1$, we denote $\mathbf{e}_{j}$ the unit vector in $\mathbb{R}^{m+1}$, whose $j^{\text {th }}$ coordinate is 1 . Let $\mathbf{u}_{0}=\mathbf{e}_{1}-\mathbf{e}_{m+1}$, and $\mathbf{u}_{j}=\mathbf{e}_{j+1}-\mathbf{e}_{j}$ for $1 \leq j \leq m$. It can be checked that the extreme rays of the normal cone at $F_{J}$ are $\mathbf{u}_{j}, 0 \leq j \leq m, j \notin J$. These vectors can be separated into $k+1$ groups: $B_{j}=\left\{\mathbf{u}_{l}: i_{j-1}<l<i_{j}\right\}, j=1,2, \ldots, k$ and $C=\left\{\mathbf{u}_{l}: l<i_{0}\right.$ or $\left.l>i_{k}\right\}$. The vectors from different groups are mutually orthogonal. Thus, $\gamma\left(N\left(F_{J}, K\right)\right)$ can be evaluated as

$$
\gamma\left(N\left(F_{J}, K\right)\right)=\gamma(C) \cdot \prod_{i=1}^{k} \gamma\left(B_{k}\right)
$$

where $\gamma(C)$ means the Gaussian measure of the cone generated by the vectors in $C$.

\section{Lemma 2}

$$
\gamma\left(B_{j}\right)=\frac{1}{i_{j}-i_{j-1}}, \quad \gamma(C)=\frac{1}{m-i_{k}+i_{0}}
$$

Proof: Add the vector $\mathbf{x}=: \mathbf{e}_{i_{j-1}+1}-\mathbf{e}_{i_{j}}$ into $B_{j}$. The expanded group contains $\left(i_{j}-i_{j-1}\right)$ vectors. Note that the sum of these vectors is $\mathbf{0}$. Thus any linear combination of these vectors can be expressed as a convex combination of $\left(i_{j}-i_{j-1}-1\right)$ vectors. In fact, for any linear combination $a \mathbf{x}+\sum_{i_{j-1}<l<i_{j}} b_{l} \mathbf{u}_{l}$, suppose $a \leq b_{l}, i_{j-1}<l<i_{j}$. Then,

$$
a \mathbf{x}+\sum_{i_{j}-1<l<i_{j}} b_{l} \mathbf{u}_{l}=a\left[\mathbf{x}+\sum_{i_{j-1}<l<i_{j}} \mathbf{u}_{l}\right]+\sum_{i_{j-1}<l<i_{j}}\left(b_{l}-a\right) \mathbf{u}_{l}=\sum_{i_{j-1}<l<i_{j}}\left(b_{l}-a\right) \mathbf{u}_{l}
$$

which is a convex combination of $\left(i_{j}-i_{j-1}-1\right)$ vectors. Because any $\left(i_{j}-i_{j-1}-1\right)$ vectors from this extended group are linearly independent, such convex combination expression is unique (except on a lower dimensional subset).

Consider the $\left(i_{j}-i_{j-1}\right)$ cones that are formed by $\left(i_{j}-i_{j-1}-1\right)$ vectors from the extended group. The argument above implies that these cones form a partition of an $\left(i_{j}-i_{j-1}-1\right)$ dimensional space. By looking at the inner products of the vectors, we notice that all these cones are reflections of one another. Therefore, they have the same measure. Hence each cone has Gaussian measure $1 /\left(i_{j}-i_{j-1}\right)$.

To compute $\gamma(C)$, we notice that the inner product structure of the vectors in $C$ is the same as the inner product structure of the vectors $\mathbf{u}_{1}, \mathbf{u}_{2}, \ldots, \mathbf{u}_{m-i_{k}+i_{0}}$. So they have the same measure. The latter has measure $1 /\left(m-i_{k}+i_{0}\right)$ by the previous argument.

Lemma 3 The $k^{\text {th }}$ intrinsic volume of $K_{n}$ is

$$
V_{k}\left(K_{n}\right)=\frac{1}{k !} \sum_{S} \sqrt{\frac{n-\left(l_{1}+l_{2}+\cdots+l_{k}\right)}{n \times l_{1} \times l_{2} \times \cdots \times l_{k}}}
$$

where $S=\left\{\left(l_{1}, l_{2}, \ldots, l_{k}\right): l_{1}+l_{2}+\cdots+l_{k} \leq m, l_{i} \geq 1,1 \leq i \leq k\right\}$. 
Proof: Applying Lemma 2 to (9), together with (8) and (7), we have

$$
V_{k}(K)=\sum_{F_{J}} \frac{1}{k !} \sqrt{\frac{n-\left(i_{k}-i_{0}\right)}{n \times\left(i_{k}-i_{k-1}\right)\left(i_{k-1}-i_{k-2}\right) \cdots\left(i_{1}-i_{0}\right)}} \frac{1}{m-i_{j}+i_{0}} .
$$

The lemma follows by changing variables: $l_{k}=i_{k}-i_{k-1}$.

Proof of Theorem 1: As $n \rightarrow \infty, m / n \rightarrow T$. By applying Lemma 3, we obtain

$$
V_{k}\left(K_{n} / \sqrt{n}\right) \rightarrow \frac{1}{k !} \int_{D} \sqrt{\frac{1-\left(x_{1}+x_{2}+\ldots+x_{k}\right)}{x_{1} x_{2} \cdots x_{k}}} d x_{1} d x_{2} \cdots d x_{k},
$$

where $D=\left\{\left(x_{1}, x_{2}, \ldots, x_{k}\right): x_{i} \geq 0 ; 1 \leq i \leq k ; \sum_{i=1}^{k} x_{i} \leq T\right\}$. By changing variables, we can express the right hand side as

$$
\frac{1}{k !} \int_{x_{1}^{2}+x_{2}^{2}+\cdots+x_{k}^{2} \leq T} \sqrt{1-\left(x_{1}^{2}+x_{2}^{2}+\cdots+x_{k}^{2}\right)} d x_{1} d x_{2} \cdots d x_{k}=\frac{1}{k !} \frac{k \omega_{k}}{2} \beta_{T}(k / 2,3 / 2),
$$

where $\beta_{T}$ is the incomplete Beta function. In particular, if $T=1$, we have $V_{k}\left(K_{n} / \sqrt{n}\right) \rightarrow \frac{\omega_{k+1}}{2 k !}$. Together with (6), this implies (4).

The proof of (5) follows from (4) and the result in [2], which states that the corresponding $k^{\text {th }}$ intrinsic volume for Brownian motion approaches $\frac{\omega_{k}}{k !}$.

Acknowledgments The author is grateful to R.A. Vitale for his interest and valuable comments. Thanks to the participants in the Probability Internship Program at the University of Wisconsin-Madison for their interest and useful conversations.

\section{References}

[1] Chevet, S. (1976). Processus Gaussiens et volumes mixtes. Z. Wahr. verw. Geb. 36, 47-65.

[2] Gao, F. and Vitale, R.A. (2001). Intrinsic volumes of the Brownian motion body, Discrete Comput. Geom. 26, 41-50.

[3] Tsirel'son, B.S. (1982). A geometric approach to maximum likelihood estimation for infinite-dimensional Gaussian location I. Theory Prob. Appl. 27, 411-418.

[4] Tsirel'son, B.S. (1985). A geometric approach to maximum likelihood estimation for infinite-dimensional Gaussian location II. Theory Prob. Appl. 30, 820-828.

[5] Tsirel'son, B.S. (1986). A geometric approach to maximum likelihood estimation for infinite-dimensional location III. Theory Prob. Appl. 31, 470-483.

[6] Vitale, R.A. (1996). The Wills functional and Gaussian processes. Ann. Probab. 24, 21722178.

[7] Vitale, R.A. (2001). Intrinsic volumes and Gaussian processes. Adv. Appl. Probab. 33, no. $2,354-364$. 